\title{
Mechanisms of crack bridging by composite and metallic rods
}

\author{
D.D.R. Cartiéa ${ }^{\mathrm{a}}$ B.N. Cox ${ }^{\mathrm{b}, *, 1}$, N.A. Fleck ${ }^{\mathrm{c}}$ \\ ${ }^{a}$ Engineering Department, Cambridge University, Cambridge, UK \\ ${ }^{\mathrm{b}}$ Rockwell Scientific Co. LLC, 1049 Camino Dos Rios, Thousand Oaks, CA 91360, USA \\ ${ }^{\mathrm{c}}$ Engineering Department, Cambridge University, Cambridge, UK
}

Received 16 September 2003; revised 24 February 2004; accepted 2 March 2004

\begin{abstract}
Experiments are reported that explore the mechanics of single pins or rods that bridge a delamination crack in a miniature model specimen. The effects of material and geometrical parameters are determined by varying the angle of the rod, its material, and the material in which it is embedded. Different tests represent mode I and mode II loading, with respect to a pre-existing delamination crack.

Many observed mechanisms are similar to those previously reported for stitches and in limited studies on rods. They include debonding and sliding of the rod relative to the substrate, lateral deflection of the rod into the substrate (when mode II is present), rod pullout, and rod rupture.

Sliding in mode I can be explained by assuming that pullout is resisted by uniform friction, which has a modest value $(\sim 1-20 \mathrm{MPa})$. However, when mode II loading is present and the rod deflects laterally, a more complicated friction behaviour is suggested. Peak load occurs well after the whole rod has begun to slide out of the specimen, implying that the pullout process is stable in mode II to large displacements. This, together with the high values observed for the peak loads and displacements suggest the presence of an enhanced friction zone (snubbing effect) extending over the segment of the rod that has been laterally deflected by mode II loading. This zone can grow under increasing shear displacements even after the whole rod begins to slide, leading to increasing shear loads (stable pullout). Various characteristics of the pullout experiments are consistent with this model.
\end{abstract}

(C) 2004 Published by Elsevier Ltd.

Keywords: B. Frachire; B. Delamination; Composites

\section{Introduction}

Through-thickness reinforcement in the form of stitches, composite and metallic rods, or woven and braided tows enhances the delamination resistance of laminates and composites [1-14]. A reasonably complete depiction of the fracture problem is now available from a combination of delamination fracture tests, especially those featuring highresolution measurements of crack displacements and theoretical models of intact through-thickness reinforcement along the crack flanks [15-19]. Through-thickness reinforcement has a profound effect on the crack tip mode ratio, tending to shut down mode I crack opening displacements [19]. Delamination arrest can persist under increasing load and failure then occurs by some other mechanism [14]. Fracture predictions must be based on

\footnotetext{
* Corresponding author. Tel.: + 1-805-373-4545; fax: + 1-805-373-4775.

1 www.rockwellscientific.com
}

large scale bridging models, in which the effect of the mixed mode tractions applied to the fracture surfaces by the through-thickness reinforcement is considered explicitly [17]. The use of $K$-formulae assuming an unbridged crack to deduce a fracture toughness value is inadequate.

Consider a delamination crack bridged by throughthickness reinforcement. The bridging traction vector, $\boldsymbol{p}$, can be expressed as a function of the jump in displacement, $2 \boldsymbol{u}$, across the crack faces. Optimal design of the throughthickness reinforcement requires knowledge of the material and geometrical factors that determine the relation $\mathbf{p}(\mathbf{u})$. Since the bridging tractions are proportional to the area density, $c_{\mathrm{s}}$, of the bridging tows, a more fundamental relation is that between the tractions, $\boldsymbol{T}$, acting over the section of a single tow on the fracture plane and $\mathbf{u}$. Following observations of the deformation of a bridging fibrous tow (stitch or fibrous rod) during delamination crack propagation, a simple model has been proposed to predict $\boldsymbol{T}(\boldsymbol{u})$ for mode II crack displacements [20]. 
This model has recently been generalised to treat fibrous bridging tows that are initially canted at an angle to the delamination fracture plane and are subjected to mixed mode crack displacements [21,22].

In the current study, the underlying assumptions of the mixed mode model and its predictions are compared with experimental data for $\boldsymbol{T}(\boldsymbol{u})$ from independent shear tests on a bridged crack, using a range of material combinations: titanium and carbon fibre rods bridging unidirectional (UD) carbon fibre/epoxy laminates and polycarbonate (PC) sheets. Prior validation of the modeling assumptions focused on data for quasi-isotropic laminates containing bridging stitches [20-22]. Tests on laminates reinforced by fibrous rods yielded only qualitative insight into mechanisms [11], rather than quantitative validation, and no good quality data for $\boldsymbol{T}(\boldsymbol{u})$ have been reported for metallic rods.

The present tests confirm the modeling assumptions of Refs. [20-22], with one major exception. For large displacements in mode II, when the entire rods begin to pull out from the laminate, the process remains mechanically stable, which can only be accounted for if the effects of snubbing, i.e. the enhancement of friction for a rod that is deflected into the laminate near the fracture plane, are included in the model. ${ }^{2}$ The enhancement is attributed to a large increase in the contact pressure on the rod when it deflects. Elaboration of the model of Refs. [20-22] to include snubbing is presented elsewhere [23]. Snubbing effects had not been manifested in prior tests on stitched laminates, since stitches are continuous and stable pullout occurs for them even in the absence of snubbing. The current tests on rods are uniquely definitive in this regard. Within the present investigation the rod orientation and the constraint against crack opening are also varied to explore the significance of mode mix.

\section{Summary of existing knowledge of mechanisms}

Detailed observations of the bridging of a delamination crack by fibrous rods have been reported for a quasiisotropic lap shear specimen [11]. The specimens contained $6-12$ rods oriented at approximately $\phi= \pm 45^{\circ}$ relative to the unit normal to the crack plane. The deformed states of rods at an inclination $\phi>0$ and $\phi<0$ are sketched in Fig. 1. The degree of deformation is defined in terms of a distribution of rotation $\theta$ along each rod. Following the terminology in Ref. [22], loading where $\theta$ and $\phi$ have

\footnotetext{
${ }^{2}$ The term snubbing in nautical affairs refers to checking a rope from sliding, e.g. by winding it around a capstan. When so wrapped, the curvature of the rope and the tension it carries raise the contact pressure between the rope and the capstan, which increases the magnitude of the friction force. Because the capstan does not deform significantly, the stress in the rope decays exponentially along the contact zone, if Coulomb friction holds. In the current problem, deformation of the substrate leads to a different stress distribution [23], but the principle is similar. The term snubbing was first applied to fibre reinforcements by Li [24].
}
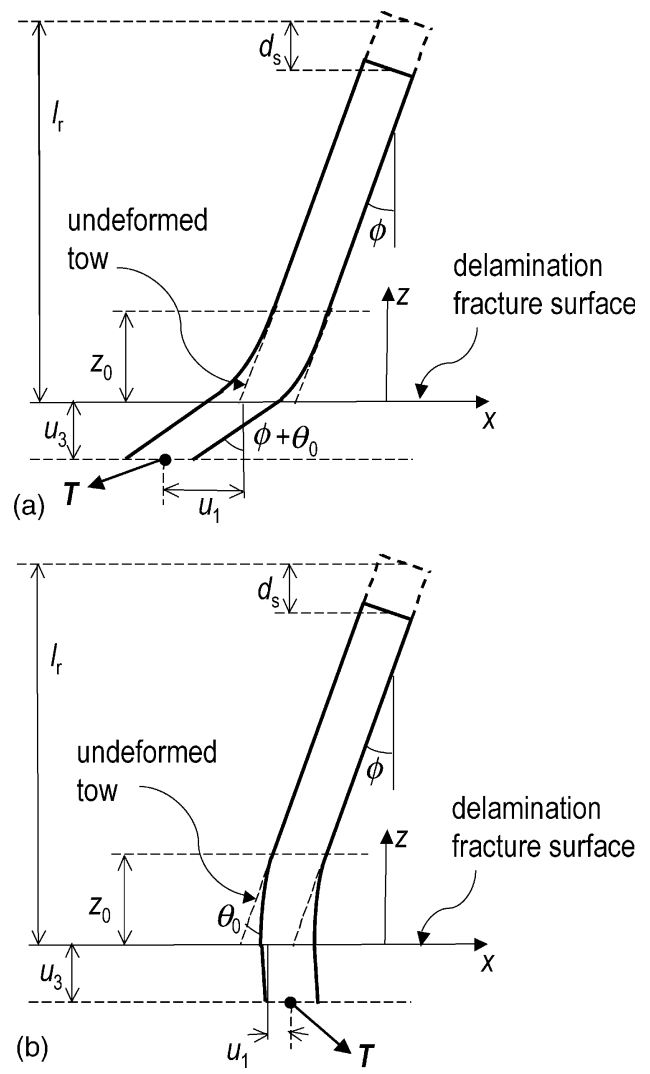

Fig. 1. Sketch of a canted tow deforming under the action of bridging tractions. A half-tow is shown, bisected at an imaginary cut upon which the bridging traction, $\boldsymbol{T}$, acts to maintain equilibrium. Cases (a) and (b) show the deformation expected when $\theta>0$ (loading with the nap) and $\theta<0$ (loading against the nap).

the same sign will be called loading 'with the nap' while loading where $\theta$ is of opposite sign to $\phi$ will be called loading 'against the nap'. It was observed that the rods deformed with the following characteristics, which were included in the model of Ref. [22].

1. The rod debonds from the laminate and pullout is resisted by friction.

2. Axial tension develops in the rods during pullout.

3. The rods shear through large strains, with matrix damage in the interior of the rod culminating in internal splitting.

4. The rods plough through the laminate. In Ref. [22] the laminate is treated as a plastic medium with a uniform and constant ploughing resistance.

The relative importance of the different mechanisms in contributing to the $\boldsymbol{T}(\boldsymbol{u})$ curve depends upon the rod's initial orientation. For rods loaded with the nap, the dominant mechanism was found to be debonding and axial sliding, culminating in rod pullout [11]. (In the initial stages of deformation, the debonded zone grows along the length of the rod and a small amount of sliding occurs between the rod and the laminate; however, the term 'pullout stage' is used here to refer specifically to sliding involving the entire 
embedded length of the rod.) Significant shear deformation tends to occur just before the pullout stage. In contrast, rods loaded against the nap exhibit little axial sliding at small delamination crack displacements, but significant shear deformation and lateral deflection through the laminate. The pullout stage begins late in the displacement history.

The damage mechanisms summarised above for fibrous rods are similar to those reported for stitches $[9,10,12]$. In stitched laminates, the stitches partially debond from the laminate when a delamination crack passes. In the presence of sliding crack displacements, the stitches plough through the laminate and deform by shear plasticity and internal splitting. However, the approach to ultimate failure is different for rods and stitches. For rods, peak load is associated either with the onset of mechanical instability during the pullout phase [23] or with rod rupture, usually at the fracture plane. For stitches, peak load occurs at stitch rupture, which generally occurs away from the fracture plane-but pullout of any remaining embedded length of stitch is immediately unstable [12].

\section{Specimens and test methods}

The traction $\boldsymbol{T}$ versus displacement $\boldsymbol{u}$ relation was measured in pure mode I tension and in approximately mode II shear by fabricating cuboidal specimens containing a pre-existing delamination along the mid-plane, which was bridged by a single rod. The specimens comprised a pair of blocks (termed hereafter 'substrates') each of dimension $10 \times 10 \mathrm{~mm}$ and thickness $2 \mathrm{~mm}$. The substrates consisted of either UD IMS/924 carbon/epoxy composite or PC. A $20 \mu \mathrm{m}$ thick PTFE release film was placed on the mid-plane of the specimen to simulate a frictionless delamination crack. Rods of diameter $0.51 \mathrm{~mm}$ were made from either commercially pure titanium or Toray T300 carbon fibreBMI resin; both types of rods were manufactured under the trade-name of Z-Fibres ${ }^{\mathrm{TM}}{ }^{3}$ Tensile tests on as-received titanium rods revealed that they had a $0.1 \%$ yield stress of $1100 \mathrm{MPa}$ and an ultimate tensile strength of $1230 \mathrm{MPa}$. The T300-BMI rods were linear elastic to failure with a strength of $1200 \mathrm{MPa}$. The rods were inserted at an angle $\phi=-40$ to $40^{\circ}$, where $\phi$ is the angle to the normal of the disbond plane (Fig. 1). If the pins were canted, they were inserted in planes that were parallel to the fibre direction in the UD laminate; and the specimens were cut so that the fibre direction would coincide with the load axis in shear tests. The sequence of manufacturing steps is as follows.

First, consider the case of UD IMS carbon-924 epoxy laminates with carbon and titanium rods. A UD Tenax IMS924 epoxy resin panel of dimension $300 \times 300 \times 4 \mathrm{~mm}$ was laid up by hand and vacuum bagged. The T300/BMI or Ti rods were supported in polystyrene foam from which they

\footnotetext{
${ }^{3}$ Z-Fibres supplied by Aztex, Inc., Waltham, Massachusetts, who own the registered trademark 'Z-Fibre'.
}

were pushed into the laminate using an ultrasonic gun (UAZ ${ }^{\mathrm{TM}}$ machine). ${ }^{4}$ The panel was then autoclaved following the manufacturer's prescription: $2 \mathrm{~h}$ at $180{ }^{\circ} \mathrm{C}$ under $0.7 \mathrm{MPa}$ pressure (7 atmosphere). Individual specimens, with a single rod in the centre, were cut from the panel using a diamond saw.

Second, consider the pinned PC specimens. Two $2 \mathrm{~mm}$ thick layers of PC sheet were fastened together with $\mathrm{Ti}$ or T300/BMI rods as follows. The titanium rods were inserted by mounting them as the bits of a high-speed drill at a speed of $1500 \mathrm{rpm}$. The rods were pushed into the PC with a force of about $1 \mathrm{~N}$ and this was facilitated by pre-chamfering the ends of the rods. Bonding was achieved by frictional melting of the PC adjacent to the titanium rod. Following drilling, the rods were left embedded in the PC. The protruding excess of rod was removed using a bench grinder. The T300/BMI rods were inserted by first predrilling the samples with a titanium rod, then withdrawing the rod and inserting the T300/BMI rod in its place.

\subsection{Tensile tests on pinned specimens}

Tensile tests were conducted on specimens for which the reinforcing rods were almost normal to the specimen midplane. The specimens were glued to T-shaped grips and the stem of each grip was then held by the jaws of the testing machine. The cross-head speed was set at $0.01 \mathrm{~mm} / \mathrm{s}$. The reported displacement is that measured at the cross-heads and therefore sums displacements across the entire specimen and loading jig assembly. Reasonable estimates show that the loading jig makes a negligible contribution, but displacements of order of magnitude $0.1 \mathrm{~mm}$ at peak load can arise, in both tensile and shear tests, from the glue used to seat the specimen halves in the loading jig.

\subsection{Shear tests}

A shear test rig was designed as shown in Fig. 2. It imposed relative, approximately shear displacements of the two pinned substrates of the laminate. The specimens were glued to the loading blocks using cyanoacrylate adhesive. Shear loading was imposed along the mid-plane of each specimen by careful control of the specimen thickness to minimize bending of the specimen.

Shear tests were conducted on specimens in which the reinforcing rod was normal or canted at an angle to the specimen mid-plane (Fig. 2). Most tests were conducted with the loading blocks free to rotate, resulting in opening displacement as well as shear displacement especially for specimens loaded against the nap $(\phi<0)$. A few tests were conducted with the specimen constrained against transverse

\footnotetext{
${ }^{4} \mathrm{UAZ}$ is an ultrasonic device for driving Z-fibres into prepreg and is supplied by Aztex, Inc., who own the registered trademark 'UAZ'. No predrilling of the laminate was required, but the T300/BMI and Ti Z-Fibres ${ }^{\mathrm{TM}}$ were given $45^{\circ}$ chamfer to aid insertion.
} 


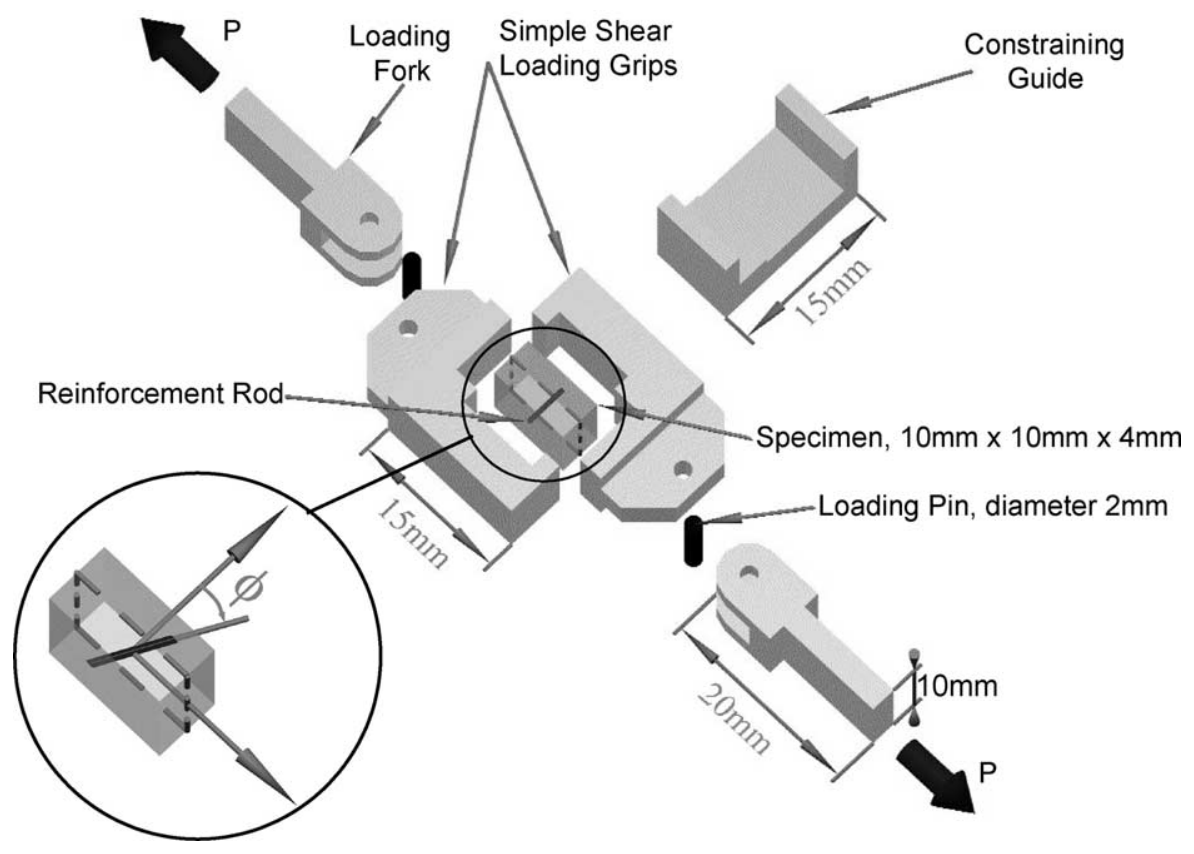

Fig. 2. Exploded view of the shear test rig.

opening using a U-section locking piece, as sketched in Fig. 2.

\section{Observed mechanisms and load/displacement data}

\subsection{Tensile tests}

Tensile tests were conducted for both Ti and T300/BMI rods in both the PC and UD laminated substrates. Typical results are shown in Fig. 3, where each curve represents a single test. In all cases, the low load levels and the shape of the load-displacement curves suggest that the rods are weakly bonded to the laminates, with relative sliding opposed by a uniform friction stress. For the UD laminated specimens, the load rose to a peak value of $40-45 \mathrm{~N}$ for both the titanium and carbon fibre rods and then dropped linearly with increasing displacement from the peak value as the rod was drawn out of the specimen (Fig. 3). The rod always pulled out from one side of the specimen, with a small amount of splitting evident in the laminate. In contrast, the interference fit between the titanium or carbon fibre rod and the PC substrate was variable, with a low pullout strength in the range of $0.2-1.5 \mathrm{~N}$ for carbon fibre rods and $1-2 \mathrm{~N}$ for Ti rods.

The friction stress opposing pullout, $\tau_{\mathrm{i}}$, can be deduced via shear lag theory from the maximum load, $F_{\max }$, and the embedded half-length, $l_{\mathrm{r}}$, of the rod that is pulled out:

$$
\tau_{\mathrm{i}}=\frac{F_{\max }}{2 l_{\mathrm{r}} \pi R}
$$

where $R$ is the rod radius. For the UD composite substrate, this relation implies $\tau_{\mathrm{i}}=15 \mathrm{MPa}$ for titanium rods and $\tau_{\mathrm{i}}=19 \mathrm{MPa}$ for T300/BMI carbon fibre rods. In contrast, for the PC substrate the inferred shear strength $\tau$ is on the order of $0.75-1.5 \mathrm{MPa}$ for titanium rods and $0.1-0.75 \mathrm{MPa}$ for the carbon fibre rods.

\subsection{Shear tests}

Shear tests were conducted for all combinations of $\mathrm{Ti}$ and carbon/epoxy rods in PC and UD laminates. The tests with unconstrained mode I opening are summarised in Fig. 4a for T300/BMI rods in PC, in Fig. 4b for titanium rods in PC, in Fig. 4c for T300/BMI rods in IMS/924, and in Fig. 4d for titanium rods in IMS/924. Results for titanium rods in IMS/924, with the specimen constrained against opening, are given in Fig. 4e for $\phi<0$ and in Fig. 4f for $\phi>0$. In Fig. 4, each curve represents a single test. The qualitative shape of the load versus displacement response showed a

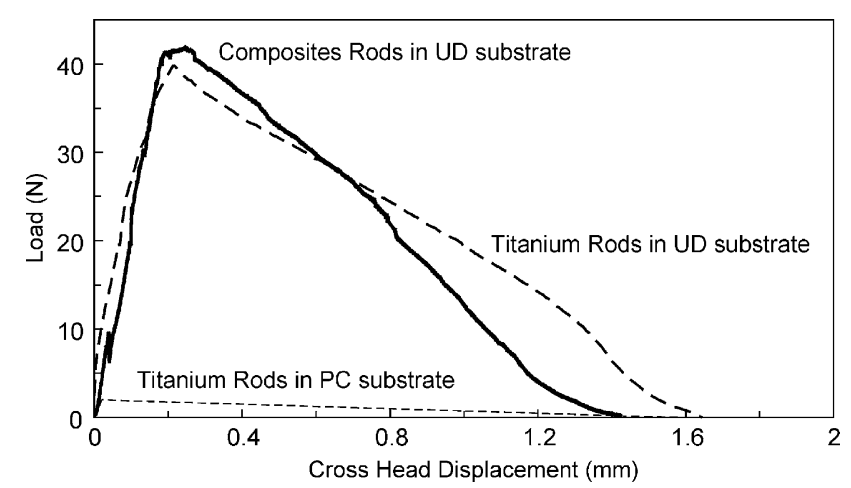

Fig. 3. Typical load-displacement data for tensile tests on T300/BMI carbon fibre and titanium rods pulled from a UD IMS/924 laminate. The pullout load for a Ti rod from a polycarbonate substrate is also sketched (complete data not recorded). 
marked dependence upon the rod type and orientation, $\phi$, and whether or not the opening displacement was constrained in the test. Consider the response of each material combination in turn.

The tests on titanium rods in PC revealed the characteristic deformation mechanisms that can be seen in Fig. 5:

(i) The rod debonds from the substrate and slides along its axis. As long as the sliding or pullout process is mechanically stable (increasing load), one would expect the specimen to remain symmetric, i.e. equal deflections of the rod in both substrate halves. The fact that one end of the rod remains largely embedded post-fracture implies that mechanical stability ceased at some point during pullout (load became a decreasing function of displacement) leading to the breaking of symmetry and the complete pullout of just one end. The remaining embedded rod presumably represents the state of both ends just prior to instability.
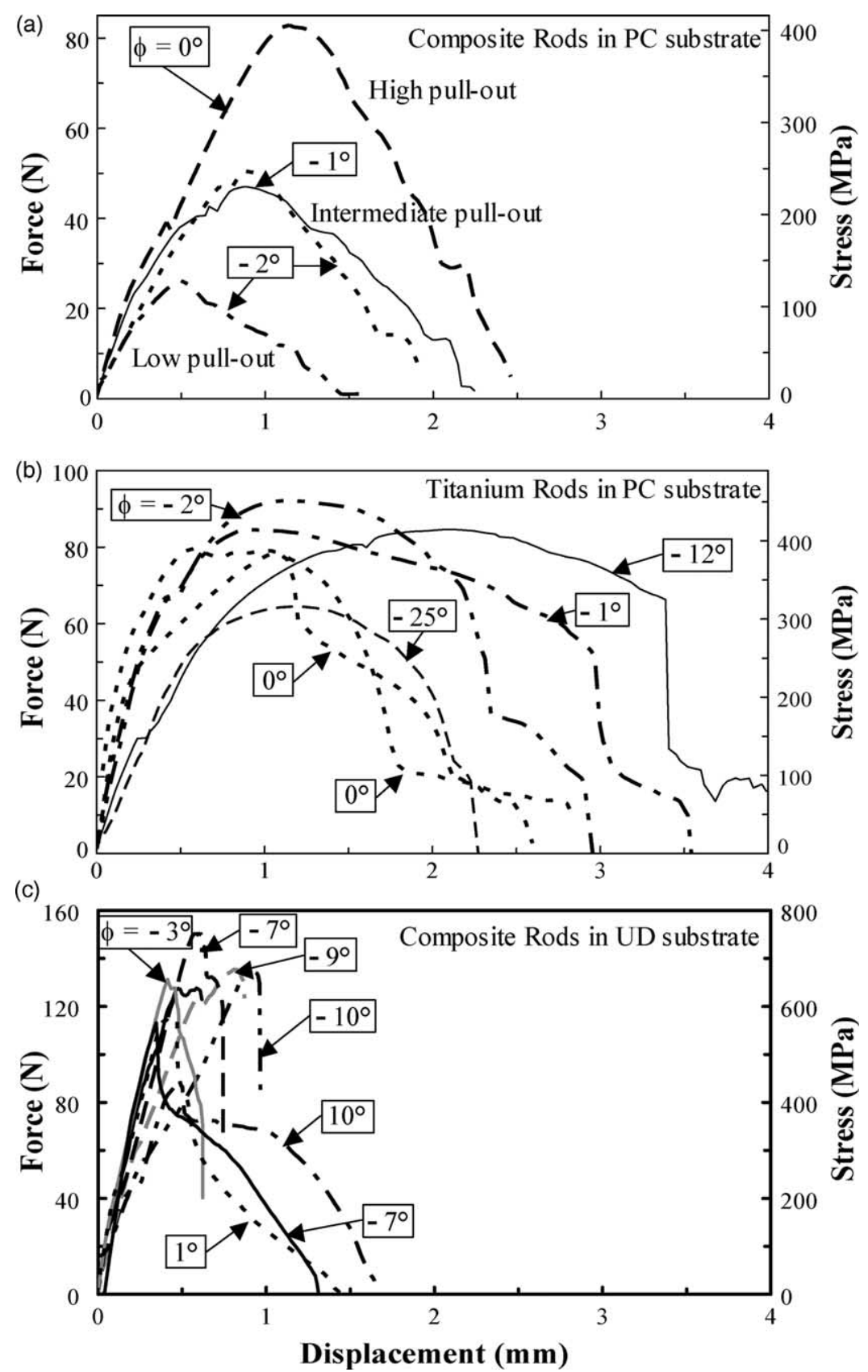

Fig. 4. Load-displacement curves from shear tests. (a) Ti rod in polycarbonate; (b) T300/BMI rod in polycarbonate; (c) T300/BMI rod in UD IMS/924 laminate; (d) Ti rod in UD IMS/924 laminate; (e) Ti rod in UD IMS/924 laminate (loading with the nap; opening constrained); (f) Ti rod in IMS/924 laminate (loading against the nap; opening constrained). 

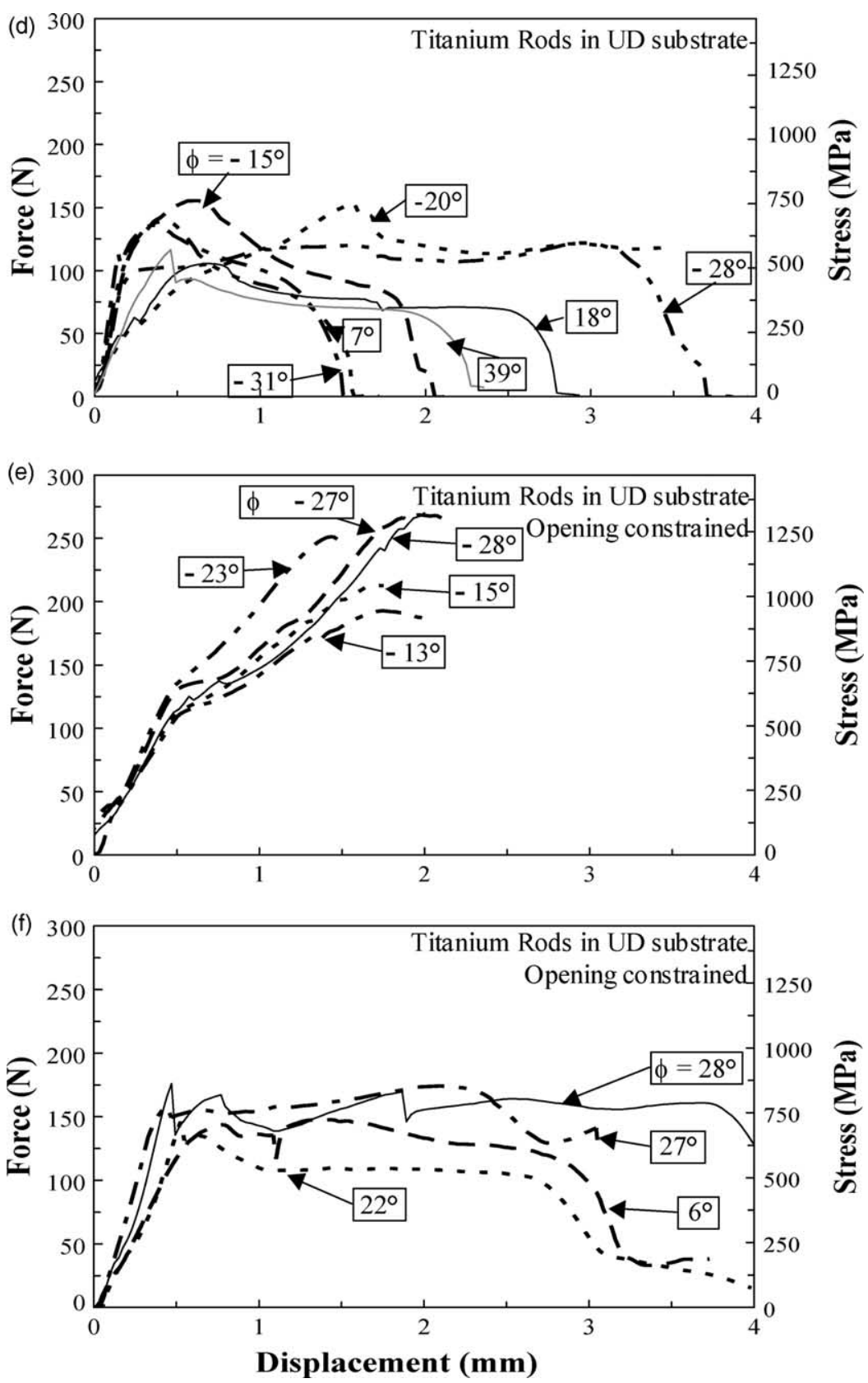

Fig. 4 (continued)

(ii) The substrate is indented plastically (with minimal cracking or loss of material) to accommodate lateral deflection of the rod.

(iii) The rod bends at two locations, one lying within the PC substrate and the other near the middle of the freestanding rod segment. The latter zone is approximately the same distance from the free-standing end as the remaining embedded length of the rod and was formed within the other substrate half prior to unstable pullout. Up to instability, symmetry implies equal pullout distances and deflection zones for both substrates. After instability commences, the stress in the rod falls and further deflection (rod bending) is

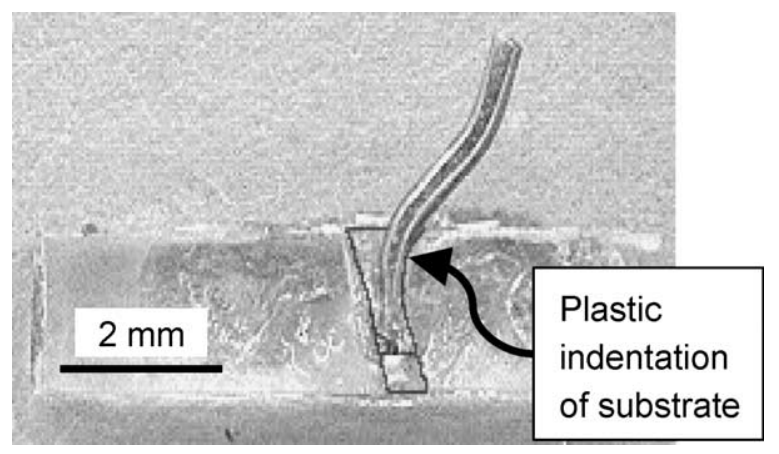

Fig. 5. Deformed Ti rod in polycarbonate following a shear test. The original locus of the rod is outlined. 


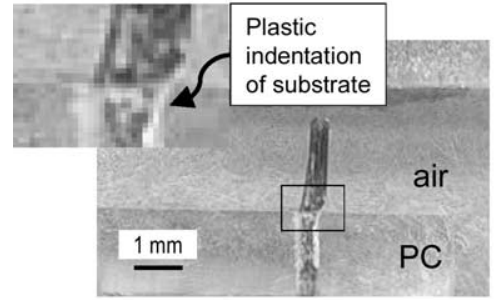

(a)

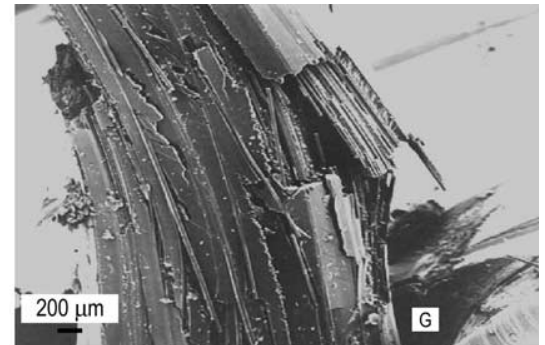

(b)

Fig. 6. (a) T300/BMI rod in polycarbonate. (b) SEM image of rod in (a) (taken from a different orientation). The inset in (a) shows an enlargement of the area indicated by the rectangle. Label $G$ in (b) shows a gap that has opened between the rod and the substrate.

limited. The segment of the rod between the two zones of bending, which lay between the fracture surfaces at the onset of instability, is almost straight. Consistently, the nearly straight segment is approximately twice as long as the distance through which the remaining embedded length has pulled out. The deformed portions of the rod are of almost uniform curvature, with a maximum transverse displacement of the rod with respect to the PC substrate of approximately $0.7 \mathrm{~mm}$.

The tests on T300/BMI rods in PC show similar mechanisms, but the length of the rod over which deflection occurs into the matrix is much less (Fig. 6). Fig. 6a (inset) shows the zone of lateral deflection, where the PC exhibits plastic deformation. The SEM micrograph in Fig. $6 \mathrm{~b}$ quantifies the lateral deflection-observe in Fig. $6 \mathrm{~b}$ the gap $G$ of width $0.2 \mathrm{~mm}$ in the PC. Fig. $6 \mathrm{~b}$ also shows that numerous splits have appeared in the composite rod, associated with shear deformation of the rod. Lower magnification micrographs show that debris associated with plastic flow of the PC has accumulated in front of the deflecting rod, but no cracking has occurred in the PC.

Fig. 7 shows Ti rods in a UD laminate matrix, after loading with and against the nap. Substantial transverse displacements (deflection) of the titanium within the UD laminate are evident, of magnitude $0.2 \mathrm{~mm}$ on the upper surface of the UD laminate for loading with the nap and $0.5 \mathrm{~mm}$ for loading against the nap. The rod pulls out before tensile rupture for loading with the nap, whereas the rod suffers a tensile necking failure before pullout for loading against the nap.
In view of these main mechanisms, the load-displacement curves for shear or mixed mode tests can be interpreted as comprising three main stages, a pre-pullout stage, a stable pullout stage, and a softening or unstable pullout stage. In the pre-pullout stage, the zone of frictional slip has not yet reached the end of the rod and, while the rod does pull out modestly, its axial displacement is limited by its elastic stiffness. The relative shear displacement between the two substrates is therefore also limited. Much greater displacements can be achieved in the stable pullout stage, which includes sliding of the embedded end of the rod almost as a rigid body [23]. If the rod ruptures during the pre-pullout or stable pullout stages, a brittle macroscopic behaviour is exhibited; the load drops sharply to zero. In contrast, if the rod does not rupture before instability, the axial stress in the rod declines gradually during unstable pullout, depending on the constraints of the test and the initial orientation of the rod.

The measured displacement sums contributions from various phenomena. When the rod pulls out without failing, the pulled out end can sustain some load right up to its departure from the substrate; and, if opening displacements are constrained (Fig. 4f) even beyond this point. Additional displacement will arise from the end of the rod that remains embedded in the other substrate, because it may slide significantly before peak load (Fig. 5). The last two significant contributions to the ultimate displacement come from lateral deflection, which might be a few tenths of a millimeter in each substrate. When all these displacement contributions are summed, the total can exceed

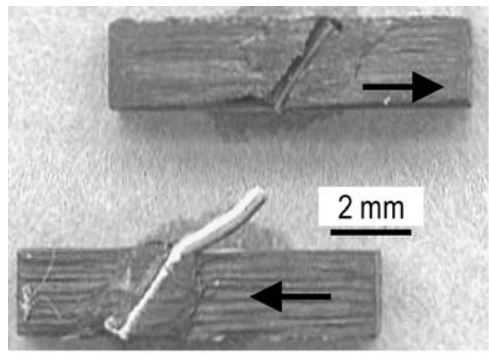

(a)

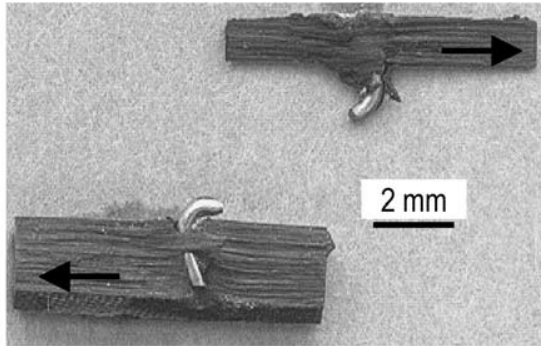

(b)

Fig. 7. Ti rods in UD IMS/924 laminate: (a) after loading with the nap and (b) after loading against the nap. The arrows indicate the direction of displacement of the two halves of the specimen. 
the half-length of the rod, especially in the case of Ti rods (Fig. 4b, d, and f).

Consider now the composite rod inserted in UD IMS/924 laminate and loaded with the nap $(\phi>0)$. Little damage is visible after the rod has pulled out of the laminate. As for titanium rods in the UD laminate, the record of load versus load point displacement shows a distinct hardening stage (pre-pullout and stable pullout), followed by a softening stage involving unstable pullout. In contrast, when the composite carbon rod is loaded against the nap $(\phi<0)$, the rods show a brittle behaviour with the rod failing in shear.

\section{Discussion}

\subsection{Enhanced friction and the stability of pullout}

A simple friction model can be constructed to account for stable pullout involving sliding of the whole rod during mode II loading. Let $\tau_{\mathrm{i}}$ denote the frictional traction observed under mode I pullout. Assume that this value of frictional traction also prevails under mixed mode pullout over the part of the rod that has not been deflected, i.e. over the more deeply embedded part of the rod. Where the rod has indented the substrate, an enhanced friction traction, $\tau_{\mathrm{e}}$, is assumed to act (which will be called a 'snubbing' effect). Both $\tau_{\mathrm{i}}$ and $\tau_{\mathrm{e}}$ are defined as averages around the circumference of the rod (they will not be uniform around the circumference, but this detail does not affect the current argument). A simple shear lag analysis of the axial stress, $\sigma_{\mathrm{z}}$, acting along the rod (averaged across the rod's section) yields the axial stress variation depicted in Fig. 8. The axial stress at the fracture plane is [23]

$\sigma_{0}=\frac{2}{R \cos \phi}\left\{\left(l_{\mathrm{r}}-d\right) \tau_{\mathrm{i}}+z_{0} \tau_{\mathrm{e}}\right\}$

where $l_{\mathrm{r}}$ is the rod's half-length, $z_{0}$ is the length of the deflection zone, and $d$ is the distance over which the end of the rod has slipped (Fig. 1). Thus

$$
\frac{\mathrm{d} \sigma_{0}}{\mathrm{~d} d}=\frac{2}{R \cos \phi}\left\{-\tau_{\mathrm{i}}+\frac{\partial z_{0}}{\partial d} \tau_{\mathrm{e}}\right\}
$$

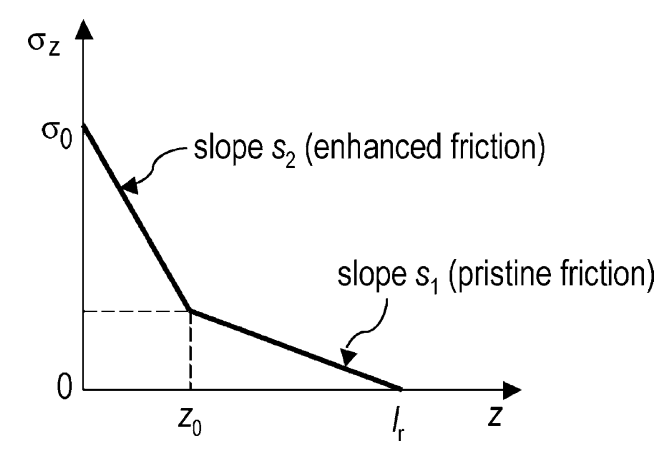

Fig. 8. Axial stress variation in a rod when friction is enhanced over the deflected zone, because of raised interfacial contact pressure. and whether $\sigma_{0}$ rises or falls as the rod pulls out ( $d$ increases) depends on the rate of increase of the length of the deflection zone. While $\sigma_{0}$ is distinct from the load applied in a test (being related to it by force resolution involving the angle of the rod), nevertheless Eq. (3) suggests a mechanism for stable pullout after the whole rod begins to slide.

The rate of increase of $z_{0}$ with increasing pullout, $d$, depends on the ratio of mode I and mode II loading and on the initial orientation of the rod. Mode II loading or loading against the nap favors large $\partial z_{0} / \partial d$, while mode I loading or loading with the nap favors small $\partial z_{0} / \partial d$. Details of the evolution of $z_{0}$ and the mechanics of the pullout process depend on the mechanics of the rod's lateral deflection, which involves deformation of both the rod and the substrate. A simple model of the whole process is presented in Ref. [23]. Stability through Eq. (3) also requires $\tau_{\mathrm{e}}>\tau_{\mathrm{i}}$. Numerical estimates, detailed below and in Ref. [23], suggest that, for the model to reproduce initially stable behaviour, $3<\tau_{\mathrm{e}} / \tau_{\mathrm{i}}<10$ in common cases.

\subsection{Ultimate load and the transition from brittle to ductile behaviour}

Fig. 9 shows the ultimate load of each experiment plotted as a function of the insertion angle $\phi$. In this figure, each point represents a single test. The scatter in the data, shown by variance from the hand fitted curves for each data set, is modest. The greatest scatter arises for composite rods inserted in $\mathrm{PC}$ in the data near $\phi \approx 0$ (open triangles); this scatter reflects the variable fit and friction that result from the imperfect manufacturing method that was used for composite rods in PC.

The ultimate load is controlled by the failure mode of the specimens, which depends on the material combination and the initial angle of the rod. The broad curve in Fig. 9, which is drawn only as a guide to the eye, separates cases of failure by fracture and failure by rod pullout. Ductile behaviour in the load-displacement record always corresponds to failure by pullout and the peak load then corresponds to the onset of instability in the pullout process. Brittle load-displacement curves correspond to rod failure and the ultimate load corresponds to the force necessary to fail the rod. Experimental observations reveal that loading against the nap tends to promote rod failure while loading with the nap tends to promote rod pullout. The transition between these cases for rods inserted in composites occurs at $\phi \approx 0$. For rods in PC, pullout occurs even for loading against the nap.

The ultimate load reached by the titanium rods in UD laminates, with constrained opening displacement, is sensitive to the rod angle (full squares in Fig. 9). In contrast, when an opening displacement is allowed, the ultimate load is insensitive to the rod angle (full diamonds).

The transition in failure mechanism can be explained tentatively by the snubbing mechanism, using shear lag estimates. Eq. (2) shows that the maximum axial stress in the rod predicted by shear lag theory in the presence of 


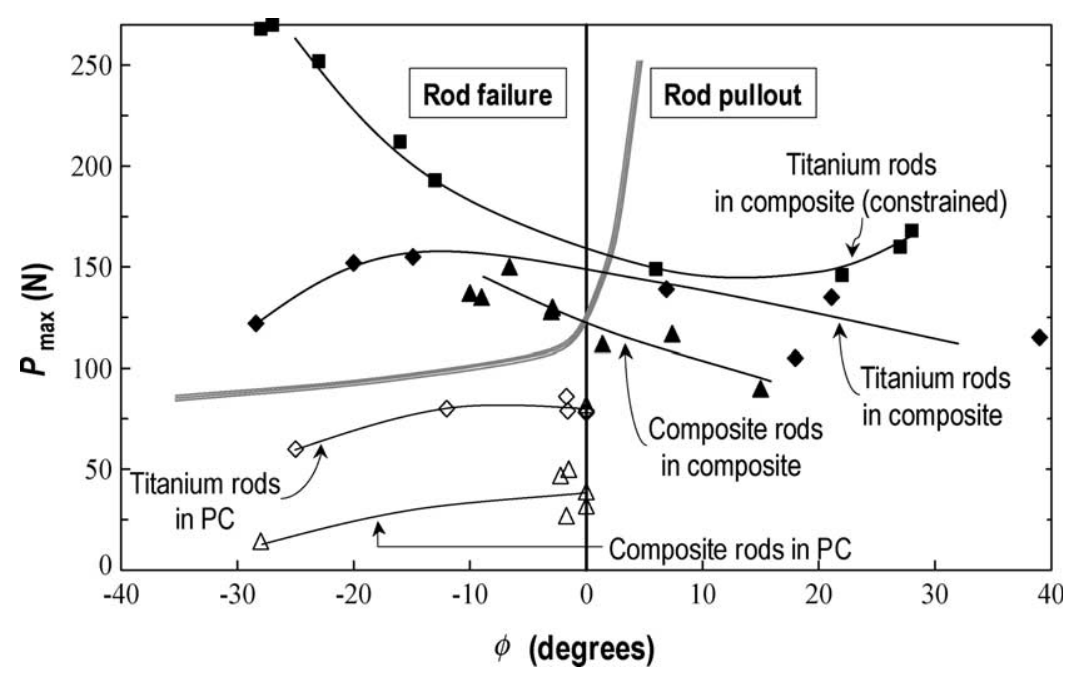

Fig. 9. Ultimate load as a function of angle $\phi$.

snubbing rises with the length of the deflected zone, $z_{0}$. For loading against the nap, the experiments show relatively large zones of deflection, whereas, under loading with the nap, the main forces acting on the rod are more aligned with its axis and the rod tends to pull out with minimal deflection. Numerical work in Ref. [23] shows that the variations in the maximum axial stress with the angle, $\phi$, can span a factor of 2-3 for common material parameters and $-\pi / 4<\phi<$ $\pi / 4$. This is easily enough to promote the transitions observed in Fig. 9, but a quantitative account requires detailed treatment of the snubbing contribution to Eq. (2), for example, using the model of Ref. [23].

\subsection{Value of the initial friction and the ductile/brittle transition}

Eq. (2) shows that the maximum axial stress in a rod will rise if the initial friction stress, $\tau_{\mathrm{i}}$, rises. Not only will the term that depends explicitly on $\tau_{\mathrm{i}}$ rise, but the length of the deflected zone will also tend to rise, since higher pristine friction tends to anchor the rod and permit greater deflection deformation, provided the rod does not fail. Thus the magnitude of the initial frictional force can have a strong effect on whether ductile or brittle behaviour is observed.

An illustration of this effect is found in the tests shown in Fig. $4 \mathrm{~b}$ and $\mathrm{c}$, which refer to specimens comprising fibrous rods in different substrates (PC versus UD carbon/epoxy laminate). The tests in PC show ductile behaviour, with distinct hardening and softening stages; in contrast, the tests in the laminate show brittle behaviour, with the hardening stage terminating in rod rupture. For the initial friction traction in these materials, mode I tests yield $\tau_{\mathrm{i}}=19 \mathrm{MPa}$ for the UD composite and $\tau_{\mathrm{i}}<0.75 \mathrm{MPa}$ for the PC. Eq. (2) implies a large difference in the maximum axial stress that can be attained for the two systems in mixed mode or predominantly mode II loading.

\subsection{Estimate of the enhanced frictional traction, $\tau_{\mathrm{e}}$}

Fig. 7b shows the state of pullout following the rupture of a $\mathrm{Ti}$ rod embedded in a UD laminate specimen. Approximate measurements reveal that, at rupture, the deflection zone has a length $z_{0} \approx 0.5 \mathrm{~mm}$; and the pullout distance $d \approx 0.33 \mathrm{~mm}$. Assume that at rupture the rod bore an axial stress, $\sigma_{0}$, equal to the peak force measured in the experiment $(\approx 150 \mathrm{MPa}$ from Fig. 4d) divided by the rod area, $0.2 \mathrm{~mm}^{2}$, i.e. $\sigma_{0}=735 \mathrm{MPa}$. (This estimate of $\sigma_{0}$ assumes that the rod is bent to be approximately parallel to the load axis at failure (Fig. 7b).) Substituting these values in Eq. (2) along with the measured value of the initial frictional stress, $\tau_{\mathrm{i}}=15 \mathrm{MPa}$, and the rod radius and length, $R=0.25 \mathrm{~mm}$ and $l_{\mathrm{r}}=2 \mathrm{~mm}$, yields $\tau_{\mathrm{e}} \approx 115 \mathrm{MPa}$. Snubbing has increased the frictional stress by a factor of $115 / 15=7.7$.

The simple model developed in Ref. [23] was also used in that work to fit data similar to those in Fig. 4c for a mode II test. In the test, a T300/BMI rod was pulled out of a UD composite laminate without rupturing. The ultimate load, which marks the onset of instability in the pullout process, and the displacement at ultimate load are sensitive to the level of enhancement of friction [23], which could therefore be determined from the stable part of the loading curve by a fitting process. The inferred enhancement factor, $\tau_{\mathrm{e}} / \tau_{\mathrm{i}}$, from the initial frictional stress, $\tau_{\mathrm{i}}=19 \mathrm{MPa}$, was in the range 6.7-9.6..$^{5}$ While the rod materials are different, there is some appeal in the fact that two different characteristics of

\footnotetext{
${ }^{5}$ In the model of Ref. [23], the relation between the shear traction in the rod resolved on the fracture plane, $T_{1}$, and the axial stress in the rod at the fracture plane, $\sigma_{0}$, is influenced by the transverse stress in the rod. Since the transverse stress can only be determined accurately by a complicated threedimensional simulation, its role is introduced in the model via a parameter, $\chi$, which has a range of feasible values. The corresponding range of inferred values of the enhancement factor for the frictional traction is that indicated in the text.
} 
pullout (load at rupture and load and displacement at loss of stability) imply similar snubbing effects.

The enhanced frictional stress, $\tau_{\mathrm{e}}$, thus has inferred values in the range $110-190 \mathrm{MPa}$. The lower end of this range, which is favoured by the value inferred from the rod rupture test and is also the most likely end of the range inferred from load/displacement fitting [23], is comparable to the expected shear strength of the UD substrate.

\subsection{Characteristics of the pre-pullout and stable pullout stages}

Fig. 4b shows that the structural stiffness of the sheared specimens with a titanium rod and a PC substrate is higher when the rod is normal to the shearing direction than when it is initially canted so as to be loaded against the nap. The initial slope of the curves for $\phi=-12$ and $-25^{\circ}$ is $30 \%$ lower than that for the curves for $\phi \approx 0^{\circ}$. For tests with titanium rods in carbon/epoxy laminates and the opening displacement constrained (Fig. $4 \mathrm{e}$ and f), the same trend is again noted. The initial slope up to about $120 \mathrm{MPa}$ is $30 \%$ lower for loading against the nap (averaged over the three cases $\phi \approx-23,-27$, and $-28^{\circ}$ ) than loading with the nap (averaged over the three cases $\phi \approx+22,27$, and $28^{\circ}$ ). The trend is not visible in Fig. $4 \mathrm{a}$ and c, where there is little variation in $\phi$, or in Fig. $4 \mathrm{~d}$, where the data appear to be relatively noisy.

An intuitive explanation of this trend is as follows. When mode II loading occurs against the nap, relatively large lateral deflections of the rod are implied by geometry, which occur against the resistance of the substrate to plastic deformation. Both the PC and the matrix in the UD laminate are relatively compliant and therefore the load-displacement curves show relatively soft behaviour. For mode II loading with the nap, the load is better aligned with the axis of the rod and relatively little lateral deflection occurs. The displacement arises more from elastic axial extension of the rod (pre-pullout regime) and sliding of the rod along its length against interfacial friction (stable pullout regime).

\subsection{Mode II pullout}

The results presented in Fig. 4a for $\phi \approx 0$ can be used to determine the ratio of the friction stress, $\tau_{\mathrm{i}}$, for the three classes of specimen. The model of Ref. [22] provides an analytical result for the relation between the applied shear traction and the shear displacement when the rod is initially normal to the fracture plane $(\phi=0)$ and the opening displacement is small. These conditions are met in the tests of Fig. $4 \mathrm{~b}$, during the early hardening stage of the curves. From Eq. (27) of Ref. [22], the term in the shear load, $F$, that depends on the initial friction traction, $\tau_{\mathrm{i}}$, is given by

$F=2\left[\frac{\pi^{2} R^{3} \tau_{\mathrm{i}} E_{\mathrm{r}} P_{\mathrm{n}}^{3}}{3}\right]^{1 / 5} u_{1}^{4 / 5}$

where $E_{\mathrm{r}}$ is the axial stiffness of the $\operatorname{rod}$ and $P_{\mathrm{n}}$ is the force per unit length of the rod by which the matrix resists the rod's deflection. This result was obtained without consideration of snubbing. Although some deflection occurred in the tests of Fig. $4 \mathrm{~b}$ and therefore snubbing effects are expected, analysis shows that, to first order in the angle of deflection, i.e. for relatively small displacements, simple force-displacement models with and without snubbing are identical [23]. Therefore, Eq. (4) should give insight into the initial slopes in Fig. 4b.

The only material parameter that varies in Eq. (4) for the tests of Fig. $4 \mathrm{~b}$ is the friction constant, $\tau_{\mathrm{i}}$. Eq. (4) shows that $F$ scales with $\tau_{\mathrm{i}}^{1 / 5}$. While $\tau_{\mathrm{i}}$ was not well measured for these specimens, relative values can be estimated from the peak loads in Fig. 4a, using Eq. (2) and assuming $z_{0}=0$, in which case the peak load $\propto \tau_{\mathrm{i}}$. The ratios of the friction stresses for the 'high-pullout,' 'intermediate-pullout,' and 'low-pullout' cases can therefore be inferred from the ratios of the peak loads, i.e. 83:47:27. Eq. (4) then predicts that the slopes in Fig. $4 \mathrm{~b}$ for small displacements should be in the proportions $1.25: 1.12: 1$. This is consistent with the data for displacements up to $0.5 \mathrm{~mm}$.

\subsection{Spatial variation of the deflection amplitude}

Fig. 10 shows a plot of the angle of deflection of the Ti rod of Fig. 5 as a function of the distance, $z$, from the fracture plane (top surface of the specimen in the figure). The variation is linear to within the accuracy of the numerical differentiation. The simplest and therefore most useful results of the models of Refs. [20-23] follow by assuming a linear variation. For friction and geometrical parameters similar to those reported here, the model of Ref. [23], when fitted to data for T300/BMI rods that pull out of UD composite substrates without rupture, predicts that the deflection zone will have a length in the range

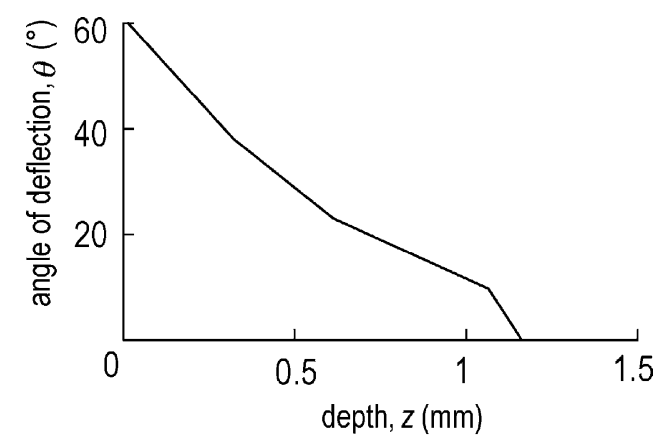

Fig. 10. Variation of the deflection angle with depth along the Ti rod of Fig. 5. 
$0.3-0.5 \mathrm{~mm}$ at peak load. This is in good agreement with the measured value of $0.5 \mathrm{~mm}$.

\section{Concluding remarks}

Titanium and composites rods have been inserted into PC and UD composite laminates. These rods have then been subjected to tensile (mode I) pullout tests and shear (mode II) tests to observe and quantify their mechanisms of deformation and failure when bridging a pre-existing delamination crack. The mode I tests, in which the rods were nominally normal to the fracture plane, yield similar results to the familiar problem of pulling a single fibre of high-aspect ratio out of a substrate. The mechanics are dominated by debonding and rod/substrate friction. The mode II tests show a much richer set of mechanisms, involving debonding, friction, substrate plasticity, and lateral deformation of the rod.

The qualitative nature of the load versus displacement response shows a marked dependence on the rod type and orientation and substrate material. Typically, rods in composite substrates loaded against the nap show brittle behaviour, with the hardening stage leading to failure of the rod by bending or shear. In contrast, rods loaded with the nap show ductile behaviour, the hardening stage being followed by a gradual unloading while the rods are pulled out of the laminate. Rods in PC substrates always exhibit ductile behaviour (rod pullout without failure).

A consideration of the displacements achieved at peak load under mode II loading shows that the process of pulling the rod out of the substrate remains mechanically stable, even when sliding occurs along the whole length of the rod. A simple explanation of this phenomenon can be found in the assumption that friction is enhanced along the zone of deflection above its pristine value, i.e. the value measured in a mode I test. Eventual loss of mechanical stability occurs at the measured peak load. Estimates imply an enhancement of friction by a factor of 3-10 for a range of common cases and about 7-10 for the particular cases of Ti or fibrous rods in a UD laminate. The enhanced frictional tractions are comparable to the shear strength of the substrate.

The effects of snubbing were not evident in prior experiments on stitches. Since the evidence for snubbing is inferred mainly from the observation of stable pullout, experiments on stitches could not reveal it, because an unbroken stitch must pull out in a mechanically stable manner by virtue of being continuous. Prior experiments on rods gave insufficient detail.

The mechanics of snubbing are taken up in Ref. [23] with a set of simplifying model assumptions that are consistent with the observations and data reported here. One important result in Ref. [23] is that the snubbing effects are strong only when the displacements become large. The initial phase of the force-displacement curve is identical for models with and without snubbing. For common parameters, snubbing effects come into play when the maximum rotation of the rods is $\sim 10^{\circ}$ [23].

Pure mode II displacements can only be achieved by mechanically constraining the specimens to avoid opening displacements. Otherwise, nominally shear tests generate some opening displacement, resulting in mixed mode conditions. Shear crack problems in engineering structures are always likely to be mixed mode, since the degree of constraint necessary to suppress opening would be unlikely to exist out of the laboratory.

\section{Acknowledgements}

BNC was supported by the US Department of Energy, grant number DE-FG03-97ER45667. Funding by the US Department of Energy does not constitute an endorsement of the views expressed in this paper.

\section{References}

[1] Horton RE, McCarty JE. Damage tolerance of composites. In: Engineered materials handbook. Vol. 1, Composites. Metals Park, OH: ASM International; 1987.

[2] Smith PJ, Wilson RD. Damage tolerant composite wing panels for transport aircraft. Boeing Commercial Airplane Company, NASA Contractor Report 3951; 1985.

[3] Dow MB, Smith DL. Damage tolerant composite material produced by stitching carbon fabrics. International SAMPE Technical Conference Series, vol. 21.; 1989. p. 595-605.

[4] Dransfield K, Baillie C, Mai Y-W. Improving the delamination resistance of CFRP by stitching-a review. Compos Sci Technol 1994; 50:305-17.

[5] Darbyshire HF, Bendix Aerospace-Electronics Company Report BDX-613-144; 1970.

[6] Bradshaw FJ, Dorey G, Sidey GR, Impact resistance of carbon fiber reinforced plastics. Royal Aircraft Establishment Technical Report 72240, Farnborough, England; 1973.

[7] Krasnov VI, Kuznetsov VA, Maksakov AYu. Automated method of transverse reinforcement of composites by short fibers. Mekhanika Kompozitnykh Materialov 1987;3:449-504.

[8] Freitas G, Fusco T, Campbell T, Harris J, Rosenberg S. Z-fiber technology and products for enhancing composite design. AGARD Conference; 1996.

[9] Mouritz AP, Jain LK. Interlaminar fracture properties of stitched fibreglass composites. In: Scott ML, editor. Proceedings 11th International Conference Composite Materials, Gold Coast, Australia. Lancaster, PA: Technomic Publishing; 1997.

[10] Cox BN, Massabò R, Mumm DR, Turrettini A, Kedward K. Delamination fracture in the presence of through-thickness reinforcement. In: Scott ML, editor. plenary paper in Proceedings 11th International Conference Composite Materials, Gold Coast, Australia, 1997. Lancaster, PA: Technomic Publishing; 1997.

[11] Rugg K, Cox BN, Ward K, Sherrick GO. Damage mechanisms for angled through-thickness rod reinforcement in carbon-epoxy laminates. Composites, Part A 1998;29A:1603-13.

[12] Turrettini A, An investigation of the mode I and mode II stitch bridging laws in stitched polymer composites, Masters Thesis, Department of Mechanical and Environmental Engineering, University of California, Santa Barbara; 1996. 
[13] Cartié DDR, Partridge IK. Z-pinned composite laminates: improvements in delamination resistance. Proc DFC5 Conf, I Mech E 1999;

[14] Rugg KL, Cox BN, Massabò R. Mixed mode delamination of polymer composite laminates reinforced through the thickness by Z-fibers. Composites 2002;A33(2):177-90.

[15] Jain LK, Mai Y-W. Analysis of stitched laminated ENF specimens for interlaminar mode-II fracture toughness. Int J Fract 1994;68(3):219-44.

[16] Jain LK, Mai Y-W. Determination of mode II delamination toughness of stitched laminated composites. Compos Sci Technol 1995;55: 241-53.

[17] Massabò R, Cox BN. Concepts for bridged mode II delamination cracks. J Mech Phys Solids 1999;47:1265-300.

[18] Massabò R, Mumm D, Cox BN. Characterizing mode II delamination cracks in stitched composites. Int J Fract 1998;92:1-38.
[19] Massabò R, Cox BN. Unusual characteristics of mixed mode delamination fracture in the presence of large scale bridging. Mech Compos Mater Struct 2001;8:61-80.

[20] Cox BN. Constitutive model for a fiber tow bridging a delamination crack. Mech Compos Mater Struct 1999;6:117-38.

[21] Cox BN. Mechanisms and models for delamination in the presence of through-thickness reinforcement. Adv Compos Lett 1999;8:249-56.

[22] Cox BN, Sridhar N. A traction law for inclined fibre tows bridging mixed mode cracks. Mech Compos Mater Struct 2002;9:299-331.

[23] Cox BN, Snubbing effects in the pullout of a fibrous rod from a laminate. Mechanics of Advanced Materials and Structures, in press.

[24] Li VC, Wang Y, Backer S. A micromechanical model of tensionsoftening and bridging toughening of short random fiber reinforced brittle matrix composites. J Mech Phys Solids 1991;39:607-25. 